\title{
Short-Term Acetylsalicylic Acid (Aspirin) Use for Pain, Fever, or Colds - Gastrointestinal Adverse Effects
} \section{A Meta-Analysis of Randomized Clinical Trials}

\author{
Angel Lanas, ${ }_{1}^{1}$ Denis McCarthy, ${ }^{2}$ Michael Voelker, ${ }^{3}$ Andreas Brueckner, ${ }^{3}$ Stephen Senn ${ }^{4}$ \\ and John A. Baron ${ }^{5}$
}

1 University of Zaragoza Medical School, Aragón Health Research Institute (IIS Aragón), CIBERehd, Zaragoza, Spain

2 Veterans Administration Medical Center and University of New Mexico School of Medicine, Albuquerque, NM, USA

3 Bayer HealthCare, Leverkusen, Germany

4 School of Mathematics and Statistics, University of Glasgow, Glasgow, UK

5 Department of Medicine, University of North Carolina, Chapel Hill, NC, USA

\section{Abstract}

Background and Aim: Acetylsalicylic acid (ASA [aspirin]) is a commonly used over-the-counter drug for the treatment of pain, fever, or colds, but data on the safety of this use are very limited. The aim of this study was to provide data on the safety of this treatment pattern, which is of interest to clinicians, regulators, and the public.

Methods: A meta-analysis of individual patient data from 67 studies sponsored by Bayer HealthCare was completed. The primary endpoints were patient-reported gastrointestinal (GI) adverse events (AEs); the secondary endpoints were the incidence of patient-reported non-GI AEs. Event incidence and odds ratios (ORs) based on Cochran-Mantel-Haenszel estimates are reported. In total, 6181 patients were treated with ASA, 3515 with placebo, 1145 with acetaminophen (paracetamol), and 754 with ibuprofen. Exposure to ASA was short term ( $82.5 \%$ of patients had a single dose).

Results: GI AEs were more frequent with ASA (9.9\%) than with placebo (9.0\%) [OR 1.3; 95\% CI 1.1, 1.5]. Dyspeptic symptoms were infrequent (4.6\% in placebo subjects). The ORs for ASA were 1.3 (95\% CI 1.1, 1.6) versus placebo; 1.55 (95\% CI 0.7, 3.3) versus ibuprofen; and 1.04 (95\% CI 0.8, 1.4) versus acetaminophen. There were very few serious GI AEs (one ASA case; three placebo cases). No differences were found for non-GI AEs and no cases of cerebral hemorrhage were reported. 
Conclusion: Short-term, mostly single-dose exposure to ASA for the treatment of pain, fever, or colds was associated with a small but significant increase in the risk of dyspepsia relative to placebo. No serious GI complications were reported.

\section{Background}

Acetylsalicylic acid (ASA [aspirin]) at doses of $\leq 325 \mathrm{mg}$ /day is used for the treatment and prevention of cardiovascular events. ${ }^{[1]}$ At higher doses, used for the treatment of pain, fever, or colds, it is one of the most commonly used over-the-counter (OTC) drugs worldwide. ${ }^{[2]}$ The recommended OTC dose for these indications varies by country, but is generally $500-1000 \mathrm{mg}$ as a single dose and 3000$4000 \mathrm{mg} /$ day. Marketing surveys indicate that treatment is usually short term for acute pain, suggesting that most people take one to two tablets for 1 day. ${ }^{[3]}$

At these doses, ASA can be considered a traditional nonselective NSAID. ${ }^{[4]}$ These compounds are associated with an increased risk of side effects, and those in the gastrointestinal (GI) tract are among the most frequently reported. ${ }^{[5]} \mathrm{GI}$ complications are life-threatening events associated with NSAID use, but other adverse effects such as dyspepsia are also important since they may lead to avoidance of treatment. ${ }^{[5,6]}$

There are only limited data on GI safety regarding short-term ASA use for the treatment of various acute conditions. Observational studies have reported the relative risks of upper GI bleeding associated with ASA above $500 \mathrm{mg}$ /day to be similar to those of other NSAIDs, but information on dose, duration of treatment, type of use, and indication is often limited or absent. Observational studies often do not differentiate between chronic and acute OTC ASA use or they do not capture OTC use at all, severely limiting the interpretation of the data. ${ }^{[7-9]}$ Moreover, data regarding more frequent GI side effects associated with NSAID use such as dyspepsia are rarely reported in the literature. ${ }^{[10]}$ Data on doses of ASA used for the treatment of pain, fever, or colds have been even less frequently reported.

Other side effects associated with ASA use include intracranial bleeding, other non-GI bleed- ing, tinnitus, dizziness, headache, impaired hearing, hypersensitivity reactions, and mental confusion. ${ }^{[11]}$ Data on the occurrence of these side effects during short-term ASA use for acute conditions have rarely been reported.

To investigate these adverse events (AEs), we evaluated the safety profile of short-term ASA use at the recommended doses for various OTC ASA indications, based on individual subject data obtained from all clinical trials with doses of ASA $\geq 325 \mathrm{mg} /$ day conducted by Bayer HealthCare between 1987 and 2008 .

\section{Methods}

\section{Setting}

Individual patient data for the meta-analysis were obtained from all studies conducted by Bayer HealthCare by 31 March 2008, where ASA was evaluated in a clinical trial setting and where adequate data documentation in terms of $\mathrm{AE}$ reporting was available. The data pool contained 87 studies in total. Among these, 33 were doubleblind, 2 were single-blind, 31 were open-label, and the blinding in one study was not recorded. All studies included were either efficacy or pharmacokinetic studies. Studies of low-dose ASA for the prevention of cardiovascular diseases (daily dose $\leq 325 \mathrm{mg}$ ) were excluded. As a result, data from 67 studies were considered for this report.

The most relevant inclusion criteria in these studies were as follows: (i) patient presented with the investigated indication in pain, fever, or colds, and was otherwise healthy (efficacy study); and (ii) volunteers (pharmacokinetic studies). The most relevant exclusion criteria were as follows: (i) history of presence of asthma or hypersensitivity to ASA, salicylate, or NSAIDs; (ii) active peptic ulcer; (iii) history of gastroduodenal bleeding; (iv) hemorrhagic diathesis; (v) impaired hepatic function; or (vi) impaired renal function. 


\section{Endpoints}

The primary endpoints were patient-reported GI AEs. During the studies, trial subjects were asked to report any AE, investigators were instructed to provide clinical diagnosis of AEs when possible, and Bayer HealthCare assigned the appropriate Medical Dictionary for Regulatory Activities (MedDRA) term to each AE. MedDRA codes (version 11.0) were used for the identification of AEs of interest in all clinical trials in the database, either according to predefined, standardized MedDRA queries or according to selected MedDRA preferred terms, high-level terms, high-level group terms, or system organ classes. The four academic authors (AL, DM, SS, JB) defined a priori the choice of terms for appropriate retrieval of these events; the same authors also defined the additional events of interest for the so-called combined preferred terms per system organ class. GI preferred terms were combined into the following variables: GI bleeding, dyspepsia, severe dyspepsia, minor dyspepsia, severe dyspepsia, any dyspepsia, abdominal pain, GI bleeding, gastroesophageal reflux disease (GERD)-related symptoms. Definitions of these combined preferred terms are included in table I.

Secondary endpoints were patient-reported nonGI AEs, including cerebral hemorrhage, other bleeding (non-GI, non-cerebral), hypersensitivity reactions, headache, dizziness, impaired hearing ability, tinnitus, mental confusion, oral complications and 'signs of overdose' as a composite of the following AEs of interest: headache, dizziness, impaired hearing ability, tinnitus, and mental confusion. MedDRA codes were also used for identification of these AEs of interest. For both primary and secondary endpoints, a time window of 7 days after drug discontinuation was used to include events from any treatment arm that were defined as 'treatment related.'

\section{Treatments Considered for the Analysis}

Clinical trials in the Bayer HealthCare database that studied treatment with ASA alone and in combination with another active ingredient (pseudoephedrine, lidocaine, or dextromethor-
Table I. Definitions of gastrointestinal (GI) combined preferred terms (PTs)

\begin{tabular}{|c|c|}
\hline$\overline{\text { Combined PT }}$ & Included PTs \\
\hline \multirow[t]{3}{*}{ Dyspepsia } & Dyspepsia \\
\hline & Epigastric discomfort \\
\hline & Eructation \\
\hline \multirow[t]{11}{*}{ Minor dyspepsia } & Abdominal discomfort \\
\hline & Dyspepsia \\
\hline & Epigastric discomfort \\
\hline & Eructation \\
\hline & Flatulence \\
\hline & Gastric dilatation \\
\hline & Gastric disorder \\
\hline & Hyperchlorhydria \\
\hline & Nausea \\
\hline & Stomach discomfort \\
\hline & Abdominal pain upper \\
\hline \multirow[t]{2}{*}{ Severe dyspepsia } & Retching \\
\hline & Vomiting \\
\hline Any dyspepsia & $\begin{array}{l}\text { Included PTs for 'Minor dyspepsia' } \\
\text { and 'Severe dyspepsia' combined }\end{array}$ \\
\hline \multirow[t]{2}{*}{ Abdominal pain } & Abdominal pain \\
\hline & Abdominal pain lower \\
\hline \multirow[t]{3}{*}{ GI bleeding } & Haematemesis \\
\hline & Haematochezia \\
\hline & Melaena \\
\hline \multirow[t]{4}{*}{ GERD-related symptoms } & Gastrooesophageal reflux disease \\
\hline & Dysphagia \\
\hline & Oesophageal pain \\
\hline & Salivary hypersecretion \\
\hline
\end{tabular}

phan) versus placebo or active comparator. For the purpose of this analysis, combinations of ASA plus vitamin C, ASA plus caffeine, and ASA plus calcium were considered to be 'ASA alone.' This study reports on comparisons between two different treatment arms: ASA versus placebo, each given alone or each combined with additional therapy (e.g. pseudoephedrine, lidocaine, or dextromethorphan). In addition, some studies were an 'active comparator.' These were acetaminophen (paracetamol), carried out comparing ASA with ibuprofen, pseudoephedrine, sumatriptan, lidocaine, ketoprofen, or, in a few cases, ergotamine tartrate and ASA-acetaminophencaffeine combinations. We report events from 
studies comparing ASA with either acetaminophen or ibuprofen, since only clinical trials with these compounds are of major clinical interest and only these studies had a sufficient number of cases to carry out appropriate analyses.

\section{Data Extraction and Management}

Data management and statistical evaluation was performed using the SAS ${ }^{\circledR}$ software package version 9.2 (SAS Institute Inc., Cary, NC, USA). The database structure was developed between Bayer HealthCare and M.A.R.C.O., Dusseldorf, Germany, an independent institute for clinical research and statistics. Data were provided by Bayer HealthCare in one of the following formats: data listings derived from medical research reports on paper, data listings from medical research reports in PDF, or SAS ${ }^{\circledR}$ datasets. Data listings provided as paper copies were entered into the database, either by independent double-data entry with subsequent comparison of the datasets and entryerror correction, or, in case of small studies with few data, by single data entry with a subsequent $100 \%$ visual inspection. Data listings provided in PDF were copied into Microsoft ${ }^{\circledR}$ Excel (Microsoft Corporation, Redmond, WA, USA) and then imported into $\mathrm{SAS}^{\circledR}$. The $\mathrm{SAS}^{\circledR}$ datasets were then transformed into the target database structure using SAS ${ }^{\circledR}$ modification programs. Information derived from the medical research reports concerning study title, design, blinding, randomization, dosing, and so forth was integrated into the target database. Appropriate quality control checks were in place at each data management step.

\section{Statistics}

The scope of the analysis, statistical methods, and content of tables and graphs was documented and pre-specified in a statistical analysis plan (SAP) by all authors prior to start of the analysis. We have estimated incidence rates for both AEs overall and those events specified by the trial investigators as adverse drug related (i.e. adverse drug reactions [ADRs]). All statistical analyses were descriptive in nature. Incidence rates calculated for this meta-analysis refer to the number of subjects who reported at least one event in the numerator and the number of all subjects under observation in the denominator.

\section{Measures of Treatment Effect}

Analysis of risk differences and odds ratios (ORs) were performed for all events of interest and treatment comparisons defined in the Treatments Considered for the Analysis section. In all analyses, trial was used as a stratum, as is standard in meta-analysis. ${ }^{[12,13]}$ These analyses were performed twice, excluding and including studies with no AEs ('zero-event studies') in both treatment arms and for all reported AEs, as well as drug-related AEs, yielding a total of 1280 analyses. Confidence interval plots (forest plots) and radial plots (Galbraith plots) were provided for the risk analyses. Estimated ORs and risk differences were based on Cochran-Mantel-Haenszel estimates, as this is robust even in 'sparse data' stratifications (i.e. where few cases of AEs occur). Weighted estimates for ORs and risk differences were calculated $^{[14-16]}$ and reported. For handling of zeroevent studies, a standardized continuity correction of 0.05 was used, taking into account the size of treatment arms. ${ }^{[16]}$ This was a deviation from the SAP, where a factor of $1 / 10000$ was proposed.

For analysis of heterogeneity over studies, a modified Breslow-Day statistic was used in the OR analyse $^{[17,18]}$ and the Cochran-Mantel-Haenszel Q-statistic was used in the risk difference analyses. ${ }^{[19]}$ A p-value of 0.10 was considered to indicate heterogeneity.

Assessment of ASA-by-subgroup interactions was carried out for sex and age. For each event of interest, a Cochran-Mantel-Haenszel risk analysis was performed and the OR for the comparisons was estimated separately between male and female subjects. The two estimates were then compared and the difference calculated (log OR [ASA vs placebo for male patients] minus log OR [ASA vs placebo for female patients]). The variance of this contrast was calculated as the sum of the variances of the two log ORs. The square root of this was taken as the standard error and used to calculate $95 \%$ confidence intervals. The test statistic for interaction was the ratio of this difference to its standard error. ${ }^{[20]}$ Analogously, estimates for the ASA-by-age interaction were derived. 
Two different subgroup allocations were considered: patients $<40$ versus patients $\geq 40$ years of age and patients $<65$ versus patients $\geq 65$ years of age. Subgroup analyses concerning daily dose, treatment duration, and treatment formulation were also performed. A p-value of $<0.10$ was assumed to provide evidence of heterogeneity.

Both treatment arms were included for parallelgroup studies; only data from the first period were included for crossover studies. Descriptive, exploratory evaluations, and statistical analyses were based on available data. AEs with incomplete or missing onset dates were considered as treatment related. The same approach was also used if no dosing date was given.

All patients treated at least once were included in the analysis. For the subgroup analyses, patients were grouped according to combinations of treatment duration (single dose, multiple dose, all) and formulation (plain: tablets, coated tablets, capsules, dry granules; effervescent tablets; intravenous; all). Multiple dosing was defined as more than one dose of study drug in any of the treatment groups.

\section{Results}

\section{Description of Trials and Demographics of Patients}

A total of 67 studies were included in the analysis (figure 1). The aim of the majority of the studies included was the evaluation of the efficacy of ASA for the treatment of pain, fever, or colds; a small number were conducted to evaluate the pharmacokinetics of ASA. Overall, exposure to

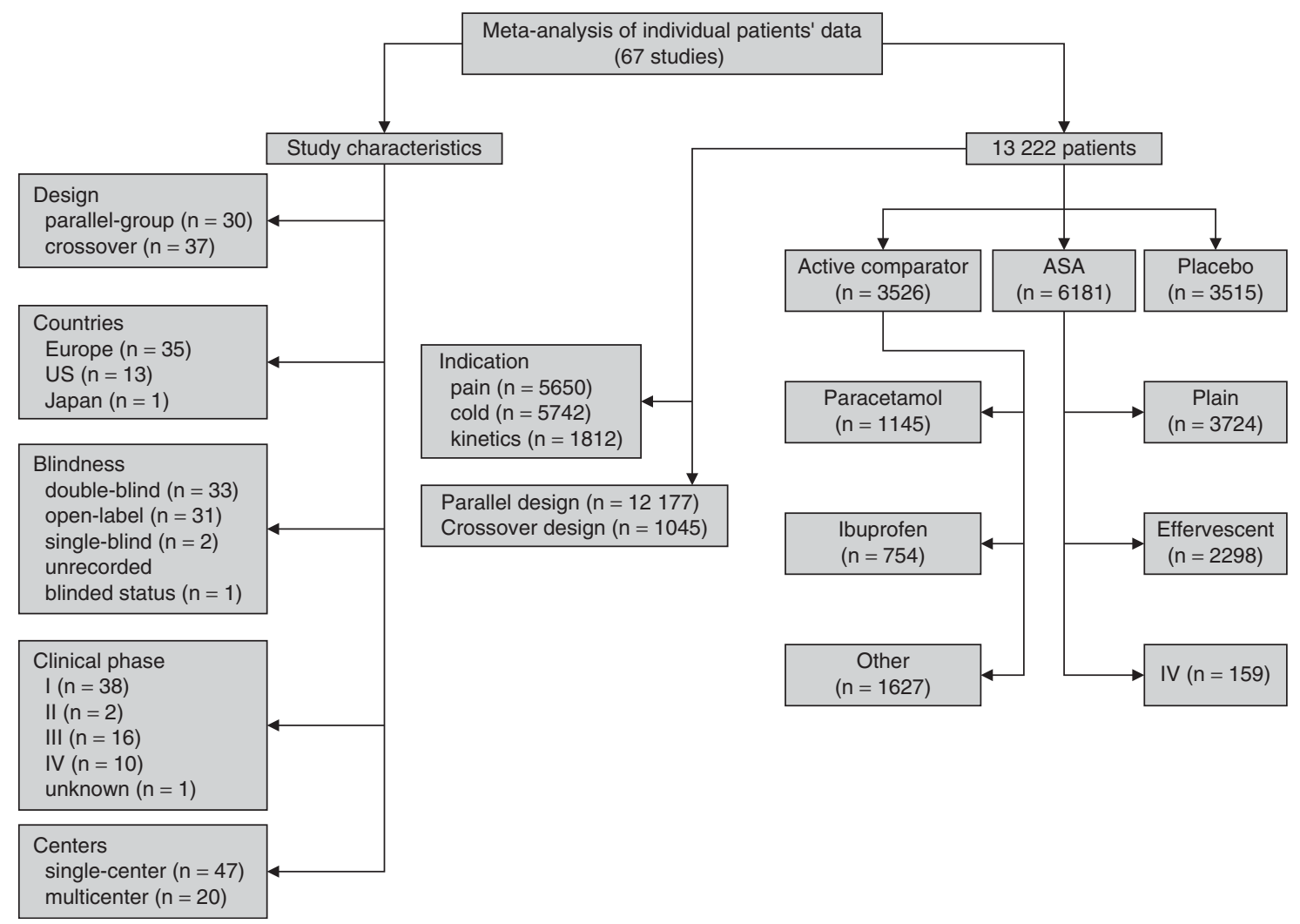

Fig. 1. Summary of the type of studies included in the meta-analysis and the distribution of patients by treatment. ASA $=$ acetylsalicylic acid (aspirin); IV = intravenous. 


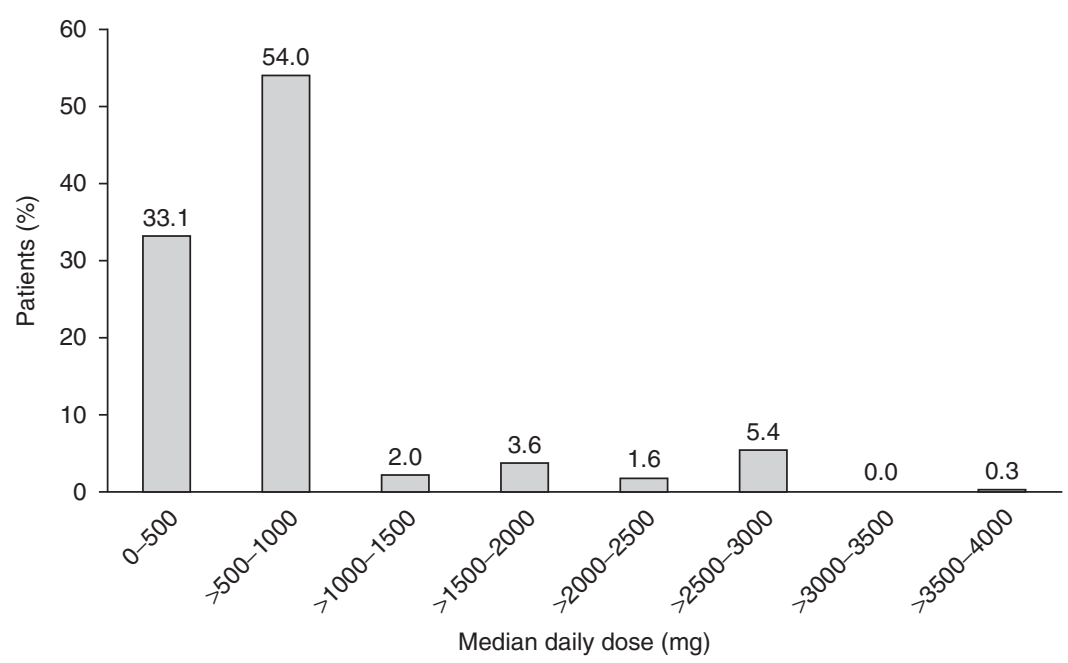

Fig. 2. Range of daily dose of acetylsalicylic acid (aspirin) taken by patients in the trials and the proportion of patients for each dose range.

ASA and its combinations was brief. Of the 6181 patients allocated to ASA and ASA combinations, $5099(82.5 \%)$ received a single dose, $1082(17.5 \%)$ received a multiple-dose regimen, and $188(3.0 \%)$ were treated for more than 5 days. Over half $(54 \%$; 3337 patients) of the ASA study population took a daily dose between 500 and $1000 \mathrm{mg}$ (figure 2). The daily doses of acetaminophen taken by patients in these trials were $300 \mathrm{mg}(31 \%), 500 \mathrm{mg}$ (16.6\%), and $1000 \mathrm{mg}(52.3 \%)$, whereas the doses of ibuprofen were $200 \mathrm{mg}(45 \%)$ and $400 \mathrm{mg}$ $(55 \%)$. The demographics of patients are included in table II.

\section{Safety Analysis}

The overall incidence of AEs was low, and was similar between subjects treated with ASA (741/ $4884 ; 15.2 \%)$ and those treated with placebo $(580 /$ $3731 ; 15.5 \%$ ) [OR $1.1 ; 95 \%$ CI $0.96,1.2$ ]; risk difference $1.0 \%(95 \% \mathrm{CI}-0.5,2.5)$. GI AEs of any severity were more frequent in the ASA groups than in the placebo groups, as summarized in table III. Risks of abdominal pain and GERDrelated symptoms were non-significantly increased in the ASA groups; severe dyspepsia was nonsignificantly less common in ASA subjects. There were very few serious GI adverse events; one case of acute appendicitis in the ASA plus pseudo- ephedrine arm and two cases in the placebo arm (one hematemesis and one appendicitis perforation). Neither the appendicitis nor the appendicitis perforation events were considered drug related by the original study investigators. GI hemorrhage (hematemesis) was reported once among subjects randomized to ASA plus dextromethorphan and three times among subjects randomized to placebo (hematemesis, hematochezia, and melena). Similar figures were observed when events were analyzed as drug-related AEs, although absolute numbers were smaller (figure 3 ).

No cerebral hemorrhage or hypersensitivity reaction occurred in any of the trials, although one occurrence of impaired hearing ability and another of mental confusion were reported in the ASA groups. Other non-GI AEs are also summarized in table III. There was a reduction in the risk of headache from ASA and there were nonsignificant increases in tinnitus and non-GI bleeding. Very similar results to those reported in table III were obtained when ASA in monotherapy was compared with placebo alone (data not shown). ${ }^{[21]}$

There was no indication of heterogeneity across studies in AEs for the OR analysis; there was statistically significant heterogeneity in the risk difference analysis for the terms GI AE, dyspepsia, minor dyspepsia, any dyspepsia, headache, signs of overdose, and oral complications. Since 
ORs were considered to be the main parameter, no steps were undertaken to correct for statistical heterogeneity in this analysis of pooled data.

Additional analyses were made according to ASA formulation, median daily ASA dose, and single- versus multiple-dose regimens. Overall, compared with plain and effervescent oral formulations, the incidence of AEs with exposure to intravenous ASA was lower (data not shown). ${ }^{[1]}$ More patients receiving doses $>1000 \mathrm{mg} /$ day reported AEs (18.1\%) than those receiving doses $<500 \mathrm{mg} /$ day $(15.1 \%)$ or those receiving placebo (13.5\%). Multiple-dose administration of ASA (up to 5 days) was associated with an increased incidence of AEs (16.2\%) when compared with single-dose administration (12.8\%), and this was similar to results observed in the placebo arm (17.0\% and $12.8 \%$, respectively).
Acetylsalicylic acid (ASA (Aspirin))-bySubgroup Interaction

Analyses for detection of an interaction between age ( $<65$ vs $\geq 65$ years of age; $<40$ vs $\geq 40$ years of age) or sex (male vs female) and the risk of experiencing an AE were carried out for the comparison of ASA versus placebo. There was no interaction by age or sex for any of the events of interest. For the combined term 'minor dyspepsia', a borderline statistically significant $(p=0.052)$ interaction effect was observed for patients $\geq 40$ years of age when compared with those $<40$ years of age (OR 1.98 [95\% CI 1.2, 3.1]). Approximately $1 \%$ of the patients were $\geq 65$ years old $(1.1 \%$ of patients on ASA, $1.3 \%$ of patients on placebo) and therefore no meaningful data can be reported for these subgroups.

Table II. Characteristics of subjects included in the meta-analysis

\begin{tabular}{|c|c|c|c|c|}
\hline Parameter & Placebo & $\begin{array}{l}\text { All acetylsalicylic acid } \\
\text { (aspirin) treatments }\end{array}$ & Active comparator & Total \\
\hline \multicolumn{5}{|l|}{$\overline{\operatorname{Sex}[n(\%)]}$} \\
\hline Men & $1257(36)$ & $2638(43)$ & $1260(36)$ & $5155(39)$ \\
\hline Women & $2258(64)$ & $3543(57)$ & $2266(64)$ & $8067(61)$ \\
\hline \multicolumn{5}{|c|}{ Race/ethnicity [n (\%)] } \\
\hline Unknown & $314(9)$ & $916(15)$ & $955(27)$ & $2185(17)$ \\
\hline Caucasian & $2917(83)$ & $4843(78)$ & $2351(67)$ & $10111(76)$ \\
\hline Black & $146(4)$ & $217(4)$ & $103(3)$ & $466(4)$ \\
\hline Asian & $27(1)$ & $41(1)$ & $20(1)$ & $88(1)$ \\
\hline Hispanic & $74(2)$ & $93(2)$ & $51(1)$ & $218(2)$ \\
\hline Other & $37(1)$ & $71(1)$ & $46(1)$ & $154(1)$ \\
\hline \multicolumn{5}{|l|}{ Age [y] } \\
\hline $\mathrm{n}$ & 3465 & 6131 & 3276 & 12872 \\
\hline Mean & 33.22 & 32.51 & 33.30 & 32.9 \\
\hline SD & 12.92 & 12.67 & 13.28 & 12.9 \\
\hline Range & $15-77$ & $15-81$ & $15-78$ & $15-81$ \\
\hline \multicolumn{5}{|c|}{ Weight [kg] } \\
\hline $\mathrm{n}$ & 3463 & 6088 & 3275 & 12826 \\
\hline Mean & 71.31 & 72.31 & 72.16 & 72.00 \\
\hline SD & 14.98 & 15.14 & 15.29 & 15.14 \\
\hline Range & $40-158$ & $35-167$ & $37-159$ & $35-167$ \\
\hline \multicolumn{5}{|c|}{ BMI $\left[\mathbf{k g} / \mathbf{m}^{2}\right]$} \\
\hline $\mathrm{n}$ & 3463 & 6088 & 3274 & 12825 \\
\hline Mean & 24.65 & 24.71 & 24.90 & 24.74 \\
\hline SD & 4.50 & 4.58 & 4.64 & 4.57 \\
\hline Range & $12.5-56.2$ & $14.5-60.6$ & $15.4-60.1$ & $12.5-60.6$ \\
\hline
\end{tabular}


Table III. Gastrointestinal (GI) adverse events (AEs) and non-GI AEs occurring in patients treated with acetylsalicylic acid (ASA [aspirin]) or placebo

\begin{tabular}{|c|c|c|c|c|}
\hline Event & ASA arm [\%] $(n=4884)$ & Placebo arm [\%] $(\mathrm{n}=3731)$ & OR $(95 \% \mathrm{Cl})$ & Risk difference $[\%](95 \% \mathrm{Cl})$ \\
\hline \multicolumn{5}{|l|}{ GI AEs } \\
\hline All GI AEs & 9.9 & 9.0 & $1.3(1.1,1.5)$ & $2.1(0.9,3.3)$ \\
\hline dyspepsia $^{a}$ & 1.8 & 1.4 & $1.7(1.2,2.4)$ & $0.8(0.2,1.3)$ \\
\hline Minor GI disorders ${ }^{b}$ & 5.0 & 4.5 & $1.2(1.0,1.5)$ & $0.9(0.0,1.8)$ \\
\hline abdominal pain ${ }^{\mathrm{c}}$ & 0.5 & 0.2 & $1.9(0.9,4.0)$ & $0.2(0.0,0.5)$ \\
\hline any dyspepsia ${ }^{c}$ & 5.3 & 4.6 & $1.3(1.1,1.6)$ & $1.2(0.3,2.2)$ \\
\hline minor dyspepsia ${ }^{c}$ & 5.0 & 4.0 & $1.4(1.1,1.8)$ & $1.5(0.6,2.3)$ \\
\hline severe dyspepsia ${ }^{c}$ & 0.6 & 0.9 & $0.7(0.4,1.2)$ & $-0.3(-0.6,0.1)$ \\
\hline GERD-related symptoms $^{c}$ & $<0.1$ & $<0.1$ & $1.5(0.3,7.0)$ & $0.03(-0.09,0.1)$ \\
\hline \multicolumn{5}{|l|}{ Non-GI AEs } \\
\hline Headache & 0.8 & 1.7 & $0.5(0.3,0.8)$ & $-0.7(-1.2,-0.2)$ \\
\hline Dizziness & 0.9 & 1.1 & $0.9(0.6,1.3)$ & $-0.1(-0.6,0.3)$ \\
\hline Tinnitus & 0.2 & 0.1 & $1.7(0.6,4.5)$ & $0.1(-0.1,0.3)$ \\
\hline Sign of overdose & 1.9 & 2.8 & $0.7(0.6,1.0)$ & $-0.6(-1.3,0.0)$ \\
\hline Other bleeding (non-GI, non cerebral) & 0.3 & 0.2 & $1.5(0.6,3.4)$ & $0.1(-0.1,0.3)$ \\
\hline Oral complications ${ }^{c}$ & 2.9 & 3.0 & $1.3(0.97,1.7)$ & $0.6(-0.1,1.3)$ \\
\hline
\end{tabular}

ASA arm $=$ ASA alone or combined with additional therapy; GERD=gastroesophageal reflux disease; MedDRA = Medical Dictionary for Regulatory Activities; $\mathbf{O R}=$ odds ratio; Placebo arm = placebo alone or combined with the additional therapy (pseudoephedrine, lidocaine, or dextromethorphan).

\section{ASA versus Active Comparator}

This analysis was carried out comparing ASA monotherapy (all doses) with acetaminophen (300-1000 mg/day) or ibuprofen (200-400 mg/day). Table IV summarizes the rates and ORs for the different GI and non-GI AEs. In general, the rates were low for all AEs. No statistically significant differences for the combined terms of dyspepsia were found between treatments, although the ORs were $>1.0$ for the comparisons with ibuprofen. Similar figures were observed when data were reported as ADRs (data not shown). ${ }^{21]}$

\section{Discussion}

This meta-analysis has shown that the risk of AEs from ASA was low at the doses and durations commonly consumed as OTC medication for the relief of pain, fever, or colds. In particular, there was a virtual absence of major GI (e.g. bleeding or perforation) and non-GI complications (e.g. cerebral hemorrhage). The use of ASA was associated with a small increase of the risk of dyspepsia when compared with placebo and with a non-significant increase in comparison with ibuprofen, another OTC drug also commonly used for the treatment of colds or acute pain. There was an increased risk of dyspepsia with ASA versus acetaminophen when considering the 'dyspepsia' MedDRA term, but not when the broader, combined dyspepsia terms defined a priori by the academic authors were used (table I).

It is important to emphasize that the conclusions obtained from this study on the safety of this therapeutic approach with ASA apply only to very short-term treatment. The majority of patients included in these trials took a single dose of $500-1000 \mathrm{mg}$ in 1 day and were relatively young. Nonetheless, the data reported are clinically valuable. First, previous information on the safety of ASA at OTC doses has relied on a small number of observational studies, since many investigations do not report on OTC use. Second, the 
data we report here are valid for typical OTC ASA use in the general population. According to some surveys on consumer use of analgesics, ${ }^{[3,22]}$ $92.4 \%$ of ASA users take one to two tablets (500 mg tablet equivalent) in a 4-week period.

Our meta-analysis agrees with two other studies reported by Edwards et al., ${ }^{[23]}$ and Steiner and Voelker. ${ }^{[24]}$ The former study was not a pooled analysis of individual patient data and was just focused on the efficacy of single-dose ASA for the relief of postoperative pain. Nevertheless, they found risks of AEs with ASA use (13\%) and placebo (11\%) similar to those reported here. They also found a significantly higher incidence of drowsiness and what they describe as 'gastric irritation,' with ASA 600-650 mg than with placebo. The latter study ${ }^{[24]}$ used pooled individual patient data from 1581 patients treated with ASA and 1271 patients treated with placebo in nine randomized, double-blind, placebo-controlled clinical trials, and these were included in our study. They were chosen because all patients used single doses of ASA $1000 \mathrm{mg}$ for the treatment of acute migraine attacks, episodic tension-type headache, and dental pain. The reported AE rates for ASA and placebo were $14.9 \%$ and $11.1 \%$, respectively; this included $5.9 \%$ of patients experiencing GI events with ASA and $3.5 \%$ experiencing them with placebo. One additional study ${ }^{[11]}$ reported side effects arising from ASA, acetaminophen, codeine, or placebo therapy in a retrospective analysis of 54 single-dose, double-blind studies involving 4346 patients with postsurgical dental pain. Both the overall and the GI AEs were low for all treatments, but codeine was associated with a higher incidence of nausea, drowsiness, and dizziness than acetaminophen, ASA, or placebo.

Available observational studies reporting on the GI safety of non-cardiovascular ASA, taken for the treatment of pain, fever, or colds, indicate that its use is associated with an increased risk of major GI complications, similar to other nonselective NSAIDs. ${ }^{[6-8]}$ In contrast, in our data and in those of Edwards et al., ${ }^{[23]}$ no serious GI complications were associated with ASA use or active comparators. This should not necessarily be seen as contradictory, since it is reasonable to assume that the populations and type of drug use studied are different. Clinical trials usually exclude patients with known GI risk factors, whereas observational studies include patients with various levels of risk. This meta-analysis probably included a population that was healthier and younger than that reported in observational studies. Furthermore, it is possible that observational studies report on patients with longer exposure to ASA use, whereas our study basically applies to those taking a single tablet or using ASA doses no higher

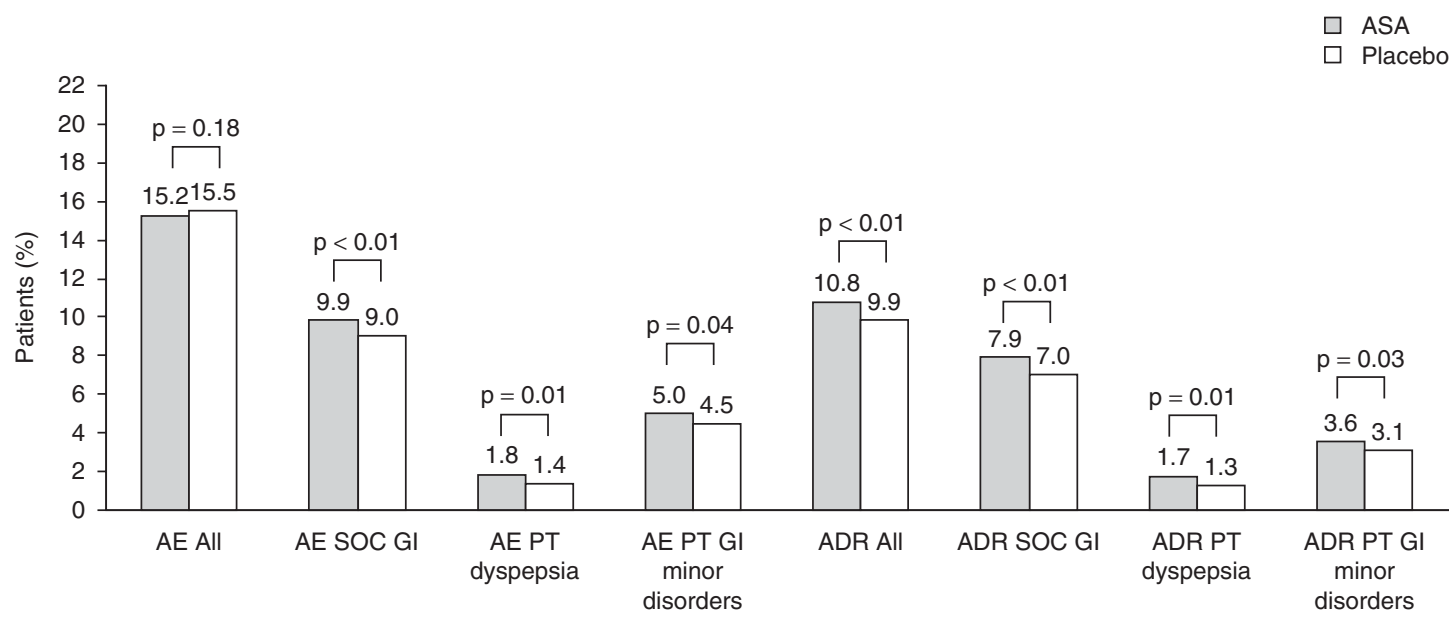

Fig. 3. Frequencies of patients who reported at least one adverse event (AE) of interest (all and drug related) with acetylsalicylic acid (ASA [aspirin]) or with placebo. $\mathbf{A D R}=$ adverse drug reaction; $\mathbf{G I}=$ gastrointestinal; $\mathbf{P T}=$ preferred term; $\mathbf{S O C}=$ system organ class. 
Table IV. Adverse events (AEs) with acetylsalicylic acid (ASA [aspirin]) in monotherapy compared with active comparators (acetaminophen [paracetamol] and ibuprofen)

\begin{tabular}{|c|c|c|c|c|c|c|}
\hline Event & $\begin{array}{l}\text { ASA [\%] } \\
(n=1128)\end{array}$ & $\begin{array}{l}\text { Acetaminophen [\%] } \\
(\mathrm{n}=1145)\end{array}$ & OR $(95 \% \mathrm{Cl})$ & $\begin{array}{l}\text { ASA [\%] } \\
(\mathrm{n}=707)\end{array}$ & $\begin{array}{l}\text { Ibuprofen [\%] } \\
(\mathrm{n}=754)\end{array}$ & OR $(95 \% \mathrm{Cl})$ \\
\hline GI AEs & 10.4 & 10.1 & $1.0(0.8,1.4)$ & 3.5 & 2.3 & $1.4(0.8,2.7)$ \\
\hline dyspepsia ${ }^{a}$ & 4.0 & 2.5 & $1.7(1.0,2.8)$ & 0.1 & 0.4 & $0.3(0.0,2.9)$ \\
\hline Minor $\mathrm{Gl}$ disorders ${ }^{\mathrm{b}}$ & 6.8 & 7.2 & $0.9(0.7,1.3)$ & 3.4 & 1.9 & $1.7(0.9,3.3)$ \\
\hline abdominal pain ${ }^{c}$ & 0.3 & $<0.1$ & $2.5(0.3,18.7)$ & 0.3 & 0.3 & $1.0(0.1,6.4)$ \\
\hline any dyspepsia ${ }^{c}$ & 7.9 & 7.6 & $1.0(0.7,1.4)$ & 2.7 & 1.6 & $1.5(0.7,3.2)$ \\
\hline minor dyspepsia ${ }^{C}$ & 7.6 & 7.2 & $1.1(0.8,1.5)$ & 2.5 & 1.3 & $1.8(0.8,3.9)$ \\
\hline severe dyspepsia $^{C}$ & 0.7 & 0.9 & $0.8(0.3,2.6)$ & 0.4 & 0.3 & $1.4(0.2,7.8)$ \\
\hline Headache & 1.0 & 1.5 & $0.6(0.3,1.49)$ & 0.7 & 0.7 & $1.0(0.3,3.4)$ \\
\hline Dizziness & 1.5 & 2.0 & $0.8(0.4,1.4)$ & 0.4 & 0.9 & $0.5(0.1,2.1)$ \\
\hline Tinnitus & $<0.1$ & 0.0 & NA & 0.3 & 0.0 & NA \\
\hline Sign of overdose & 2.6 & 3.5 & $0.7(0.4,1.2)$ & 1.4 & 1.7 & $0.8(0.3,2.0)$ \\
\hline $\begin{array}{l}\text { Other bleeding (non-GI, } \\
\text { non cerebral) }\end{array}$ & $<0.1$ & 0.2 & NA & 0.1 & 0.0 & NA \\
\hline Oral complications $^{c}$ & 0.2 & 0.8 & $0.2(0.1,1.1)$ & 0.1 & 0.1 & $1.0(0.1,12.2)$ \\
\hline
\end{tabular}

ASA arm =ASA alone or combined with additional therapy; $\mathbf{G I}=$ gastrointestinal; MedDRA=Medical Dictionary for Regulatory Activities; $\mathbf{N A}=$ not available; $\mathbf{O R}=$ odds ratio; placebo arm = placebo alone or combined with the additional therapy (pseudoephedrine, lidocaine or dextromethorphan).

than $1000 \mathrm{mg} /$ day for the relief of pain, fever, or colds. The same considerations may apply to the absence of GI bleeding events with ibuprofen in this study, where the doses used were low (200$400 \mathrm{mg}$ /day).

ASA use has been associated with other nonGI AEs, both serious and less serious. ${ }^{[11,25,26]}$ No cases of serious non-GI AEs were reported and no significant differences were found between ASA and placebo or between ASA and ibuprofen or acetaminophen for less-serious non-GI AEs in this meta-analysis.

We did not find statistically significant heterogeneity in the ORs in our analysis, suggesting that these statistics are probably generalizable, at least to the sort of patient included in the studies. However, as might be expected with relatively constant ORs and differing background rates of AEs, there was heterogeneity in the risk differences. This finding suggests that the risk differences cannot be directly applied in other clinical contexts.

The data presented in this study have several limitations. The way ASA is used may vary among different populations and therefore the data reported may not be applicable to them. The report from Curhan et al. ${ }^{[2]}$ from the Nurses' Health Study showed that ASA was used daily by $25.4 \%$ of women $>51$ years of age, and that $40.1 \%$ used ASA $\geq 1$ day/week. Since it is now widely known that ASA protects against cardiovascular events and colon cancer, ${ }^{[1,27]}$ some may take this compound very often or on a daily basis for these purposes. In any case, multiple-day exposure to ASA is not uncommon in clinical practice, and the data provided here, although focused on short-term use of ASA for the treatment of acute pain, fever, or colds, suggest an increased risk of AEs with increasing dose and multiple doses.

Another limitation of this study is that the safety data reported here may not be valid for the entire population with access to OTC ASA for the relief of pain, fever, or colds. The risk factors for GI complications in NSAID and ASA users are well defined and include older age ( $>65$ years old), patients with ulcer history, and patients with concomitant use of other NSAIDs, corticosteroids, 
or anticoagulants. Patients at risk (elderly patients or those with an ulcer history) were excluded in these clinical trials.

Finally, the very low incidence of serious AEs in our study population limited our ability to investigate differences between ASA and placebo or other comparators in this regard. Much larger studies would be required to generate enough events to make such an analysis feasible.

\section{Conclusion}

In conclusion, this meta-analysis of clinical trials of short-term ASA, mostly single dose and of 1-day duration, used for the treatment of pain, fever, or colds at common OTC doses, has shown a low incidence of AEs. There was a small increase in the risk of mild to moderate dyspepsia and abdominal pain with ASA, but no significant occurrence of major GI complications was observed.

\section{Acknowledgments}

This study was funded by Bayer HealthCare and is a metaanalysis of individual data obtained from all studies conducted by Bayer HealthCare by 31 March 2008. The database structure was based on agreement between Bayer HealthCare and M.A.R.C.O., Dusseldorf, Germany, an independent institute for clinical research and statistics. The four academic authors (AL, DM, SS, JB) defined a priori the choice of terms for appropriate retrieval of these events; the same authors also defined the additional events of interest. The academic authors had full access to all of the data in the study and they interpreted the results; the academic authors take responsibility for the integrity of the data and the accuracy of the data analysis. The academic authors wrote the manuscript but Bayer authors have also contributed to both interpretation of data and preparation and approval of the manuscript.

Angel Lanas received an honorarium from Bayer for conducting this study and preparing the manuscript. He has also consulted for Pfizer, AstraZeneca and Nicox. He has received honoraria and grants from Pfizer and AstraZeneca. Denis McCarthy, Stephen Senn and John Baron consulted for Bayer during this study. Stephen Senn received honoraria from Bayer for providing advice during this study. John Baron has previously received a grant from Bayer for a meta-analysis on aspirin. John Baron jointly holds a patent for aspirin as a chemopreventing agent for colorectal adenomas. Andreas Brueckner and Michael Voelker are employees of Bayer HealthCare.

\section{References}

1. Antithrombotic Trialists' (ATT) collaboration, Baigent C, Blackwell L, et al. Aspirin in the primary and secondary prevention of vascular disease: collaborative meta-analysis of individual participant data from randomised trials. Lancet 2009 May 30; 373 (9678): 1849-60

2. Curhan GC, Bullock AJ, Hankinson SE, et al. Frequency of use of acetaminophen, nonsteroidal anti-inflammatory drugs, and aspirin in US women. Pharmacoepidemiol Drug Saf 2002 Dec; 11 (8): 687-93

3. McNeil Consumer and Specialty Pharmaceuticals. McNeil's background package on acetaminophen: executive summary [online]. Available from URL: http://www.fda.gov/ ohrms/dockets/ac/02/briefing/3882B1_13_McNeil-Acetami nophen.htm [Accessed 2011 Apr 25]

4. Vane JR. Inhibition of prostaglandin synthesis as a mechanism of action for aspirin-like drugs. Nat New Biol 1971; 231: $232-5$

5. Fries JF, Bruce B. Rates of serious gastrointestinal events from low dose use of acetylsalicylic acid, acetaminophen, and ibuprofen in patients with osteoarthritis and rheumatoid arthritis. J Rheumatol 2003; 30 (10): 2226-33

6. Rostom A, Muir K, Dubé C, et al. Gastrointestinal safety of cyclooxygenase-2 inhibitors: a Cochrane Collaboration systematic review. Clin Gastroenterol Hepatol 2007 Jul; 5 (7): $818-28$

7. Lanas A, García-Rodríguez LA, Arroyo MT, et al. Risk of upper gastrointestinal ulcer bleeding associated with selective cyclo-oxygenase- 2 inhibitors, traditional nonaspirin non-steroidal anti-inflammatory drugs, aspirin and combinations. Gut 2006; 55 (12): 1731-8

8. Lanas A, Bajador E, Serrano P, et al. Nitrovasodilators, low-dose aspirin, other nonsteroidal antiinflammatory drugs, and the risk of upper gastrointestinal bleeding. N Engl J Med 2000; 343 (12): 834-9

9. Henry D, Lim LL, García-Rodríguez LA, et al. Variability in risk of gastrointestinal complications with individual non-steroidal anti-inflammatory drugs: results of a collaborative meta-analysis. BMJ 1996 Jun 22; 312 (7046): $1563-6$

10. Moore RA, Derry S, Makinson GT, et al. Tolerability and adverse events in clinical trials of celecoxib in osteoarthritis and rheumatoid arthritis: systematic review and metaanalysis of information from company clinical trial reports. Arthritis Res Ther 2005; 7 (3): R644-65

11. Cooper SA, Press K. Review of side effects: ten years of experience with the dental pain model. Adv Ther 1985; 2: 20-9

12. Borenstein M, Hedges LV, Higgins JP, et al. Introduction to meta-analysis. Chichester: Wiley, 2009

13. Senn SJ. The many modes of meta. Drug Inf J 2000; 34: 535-49

14. Robins J, Breslow N, Greenland S. Estimators of the MantelHaenszel variance consistent in both sparse data and largestrata Limiting models. Biometrics 1986; 42 (2): 311-23

15. Greenland S, Robins JM. Estimation of a common effect parameter from sparse follow-up data. Biometrics 1985; 41 (1): 55-68

16. Sweeting MJ, Sutton AJ, Lambert PC. What to add to nothing? Use and avoidance of continuity corrections in metaanalysis of sparse data. Stat Med 2004; 23 (9): 1351-75

17. Breslow NE, Day NE. Classical methods of analysis of grouped data. In: Statistical methods in cancer research, vol. 1. The analysis of case-control studies. Lyon: International Agency for Research on Cancer, 1980: 122-46 
18. Tarone RE. On heterogeneity tests based on efficient scores. Biometrika 1985; 72 (1): 91-5

19. Deeks JJ, Altman DG, Bradburn MJ. Statistical methods for examining heterogeneity and combining results from several studies in meta-analysis. In: Egger M, Smith GD, Altman DG, editors. Systematic reviews in health care: meta-analysis in context. London: BMJ, 2000: 285-312

20. Altman DG, Bland JM. Interaction revisited: the difference between two estimates. BMJ 2003; 326 (7382): 219

21. Data on file. Noble Shield Report. Morristown (NJ): Bayer HealthCare Consumer Care, 2010

22. Kauffman D, Kelly JP, Rosenberg L, et al. Recent patterns of medication use in the ambulatory adult population of the United States: the Slone survey. JAMA 2002; 287 (3): $337-44$

23. Edwards JE, Oldman AD, Smith LA, et al. Oral aspirin in postoperative pain: a quantitative systematic review. Pain 1999; 81 (3): 289-97
24. Steiner T, Voelker M. Gastrointestinal tolerability of aspirin and the choice of over-the-counter analgesia for shortlasting acute pain. J Clin Pharm Ther 2009; 34 (2): 177-86

25. de Weck AL, Gamboa PM, Esparza R, et al. Hypersensitivity to aspirin and other nonsteroidal anti-inflammatory drugs (NSAIDs). Curr Pharm Des 2006; 12 (26): 3347-58

26. Le Parc JM, Van Ganse E, Moore N, et al. Comparative tolerability of paracetamol, aspirin and ibuprofen for short-term analgesia in patients with musculoskeletal conditions: results in 4291 patients. Clin Rheumatol 2002; 21 (1): 28-31

27. Baron JA. Aspirin and NSAIDs for the prevention of colorectal cancer. Recent Results Cancer Res 2009; 181: 223-9

Correspondence: Professor Angel Lanas, Service of Digestive Diseases, University Hospital Lozano Blesa, C/Domingo Miral s/n, 50009 Zaragoza, Spain.

E-mail: alanas@unizar.es 